\title{
Rapid Synthesis of Large Quantities of Zinc Oxide Nanorods
}

\author{
Hu Peng ${ }^{\mathrm{a}, \mathrm{b}}$, Yuan Fangli ${ }^{\mathrm{a}}$ *, Bai Liuyang ${ }^{\mathrm{a}, \mathrm{b}}$, Li Jinlin $^{\mathrm{a}}$, Chen Yunfa $^{\mathrm{a}}$ \\ Institute of process Engineering, Chinese Academy of Sciences, Beijing 100080, China \\ ${ }^{b}$ Graduate School of the Chinese Academy of Sciences, Beijing 100049, China
}

Synthesis of basic carbonate of zinc:

Reagent grade $\mathrm{ZnSO}_{4} \cdot 7 \mathrm{H}_{2} \mathrm{O}$ and $\mathrm{NH}_{4} \mathrm{HCO}_{3}$ were dissolved in distilled water, and the concentrations were $1.5 \mathrm{~mol} / \mathrm{l}$ and $2.5 \mathrm{~mol} / \mathrm{l}$ respectively. In experiment, 2.231 of $\mathrm{ZnSO}_{4}$ solution was added to 2.551 of $\mathrm{NH}_{4} \mathrm{HCO}_{3}$ solution with continuous stirring. The reaction temperature was kept at $45 \square$. The final $\mathrm{pH}$ value of the slurry was 6.8-7.0. After precipitation of white basic carbonate of zinc, it was stirred for another 30 min to make them react completely. The slurry was then washed and filtered so as to remove $\mathrm{SO}_{4}{ }^{2-}$ ions in the filtered solution. Finally, the washed $\mathrm{BCZ}$ was oven-dried at $80 \square$. TEM image and XRD pattern of synthesized BCZ are shown in Figure S1 and Figure S2.

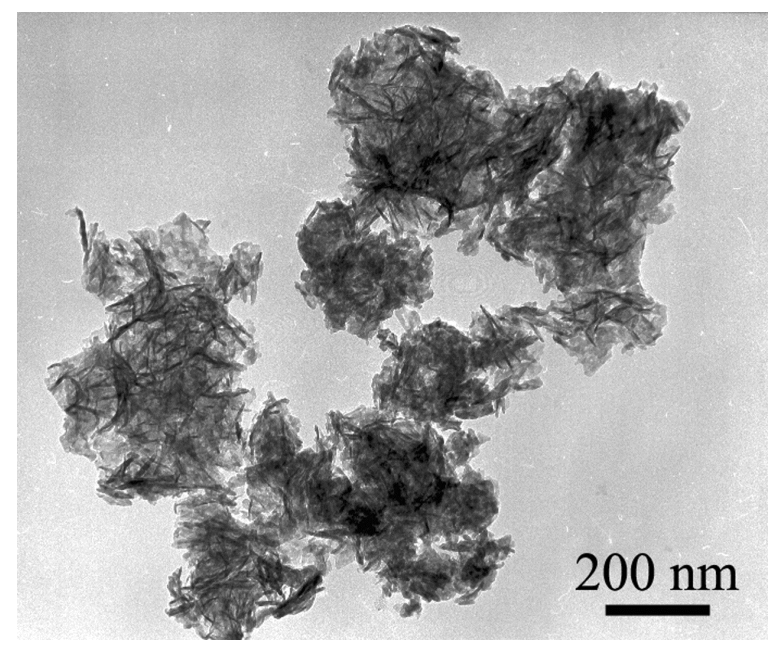

Figure S1 TEM image of as-synthesized basic carbonate of zinc.

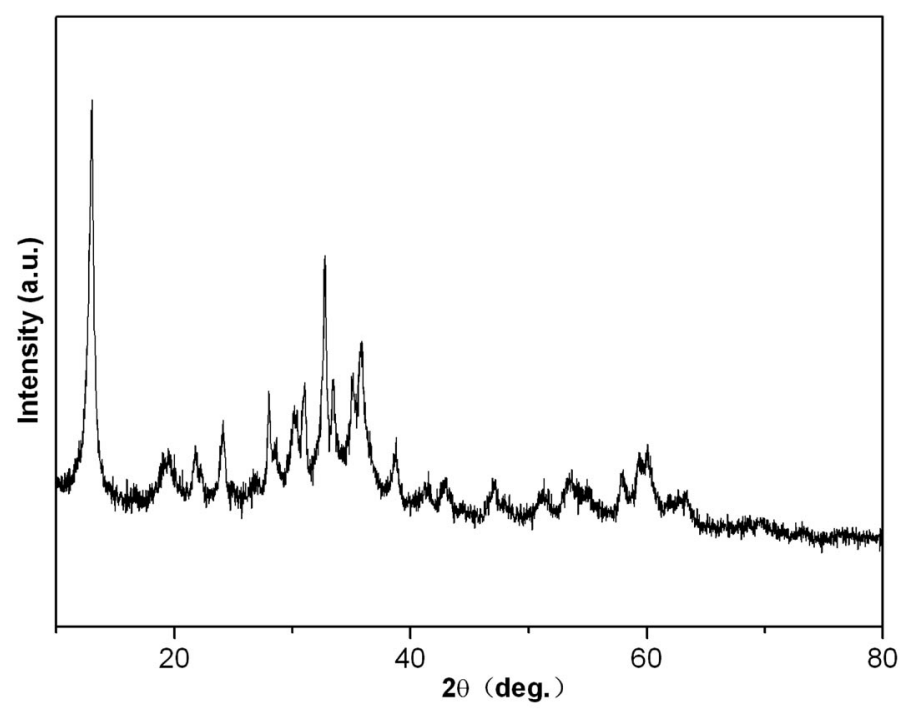

Figure S2 XRD pattern of as-synthesized basic carbonate of zinc. 
Figure S3 shows the XRD pattern of products showing in Figure 5a of the manuscript, irregular nanorods were obtained at very low oxygen rate about $0.51 / \mathrm{min}$, and the XRD analysis shows that the products were mixture of $\mathrm{Zn}$ and $\mathrm{ZnO}$.

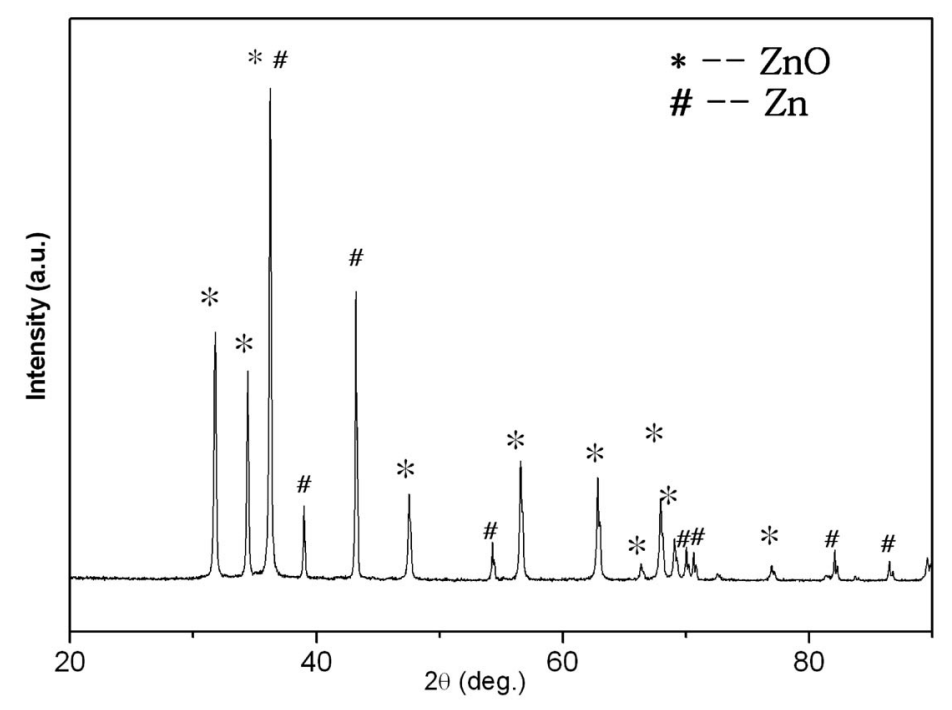

Figure S3 XRD pattern of nanorods obtained at very low oxygen rate about $0.51 / \mathrm{min}$.

\section{Synthesis of $\mathrm{ZnS}$}

$\mathrm{ZnS}$ was synthesized using the same method, staring materials of $\mathrm{Zn}$ and S(-200 mesh) were mixed firstly( mole ratio, 1:1 ). In experiment, the starting materials were supplied into the plasma flame by the carrying gas in a continuous way and then underwent vaporization, crystallization and growth processes. Figure S4 shows the TEM image and XRD of synthesized ZnS tetrapod crystals.
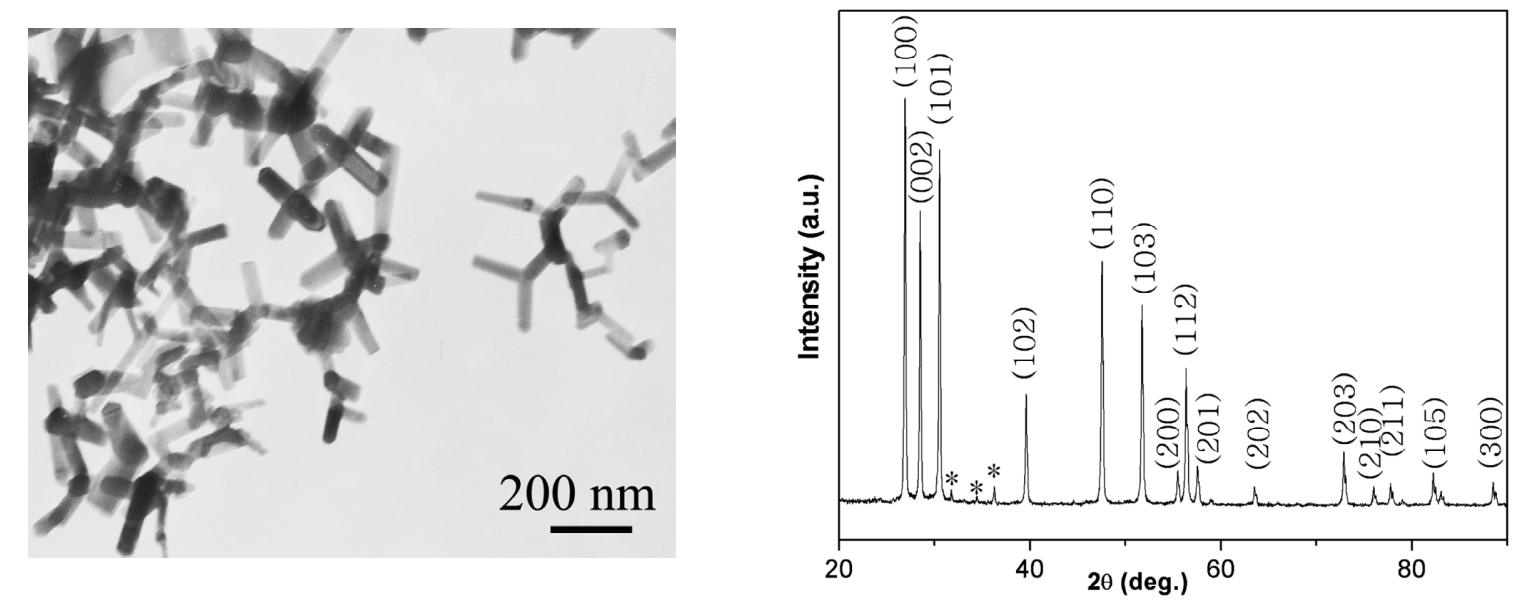

Figure S4 TEM image and XRD pattern of synthesized ZnS tetrapod.

From the XRD analysis we can see that wurtzite $\mathrm{ZnS}$ with lattice constants of $a=3.8209 \AA, c=6.2573$ $\AA$ was obtained, but there still have little amount of $\mathrm{ZnO}$ were synthesized(as marked $*$ in the XRD pattern). This is because that the nitrogen gas used in our experiment was industrial product, and 
there are about $0.5 \%$ content of oxygen gas in it. When the starting materials were supplied into the plasma flame, the reaction between zinc and oxygen is prior than zinc and $\mathrm{S}$ from the thermodynamics reason, accordingly $\mathrm{ZnO}$ synthesized is prior than $\mathrm{ZnS}$. Consequently, the products we obtained were wurtzite $\mathrm{ZnS}$ with little amount of $\mathrm{ZnO}$.

By changing the reaction condition, we can also obtain wurtzite $\mathrm{ZnS}$ nanowire and nanobelt, as shown in Figure S5.

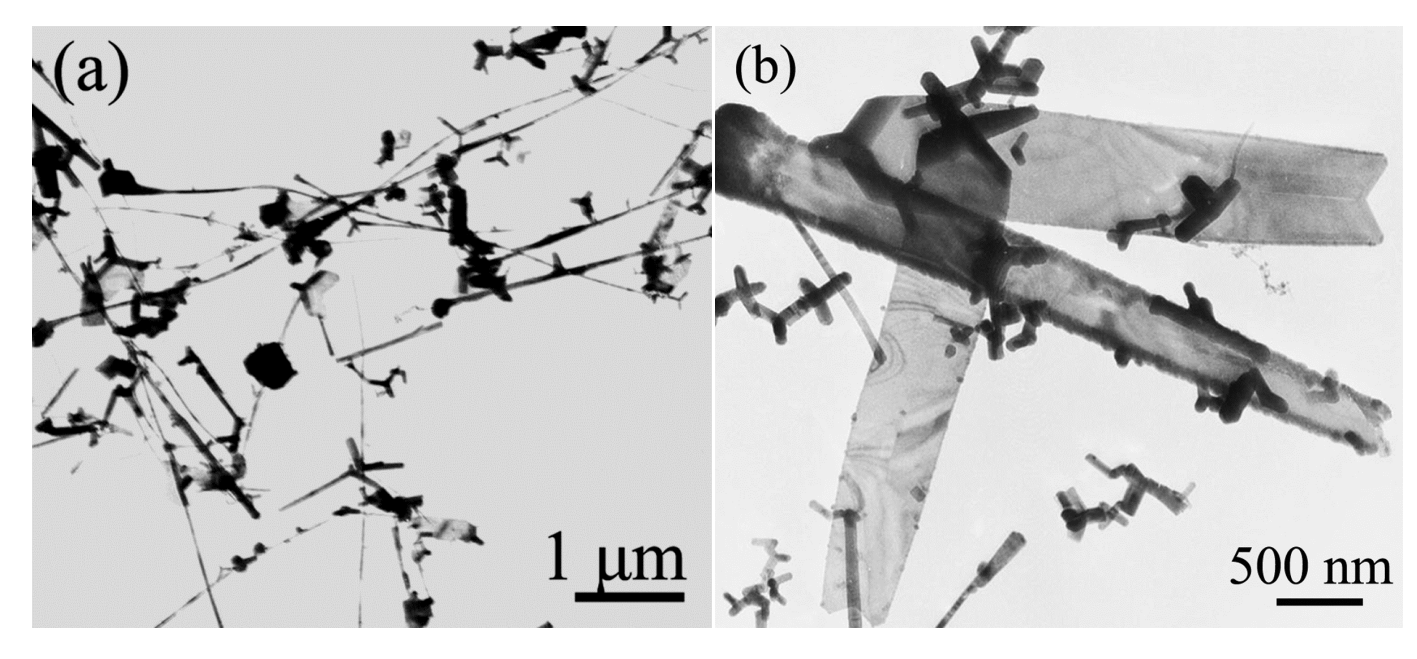

Figure S5 TEM images of synthesized ZnS nanowire and nanobelt 